\title{
Optimal Proportional Reinsurance in a Bivariate Risk Model
}

\author{
Cristina Gosio, Ester C. Lari, Marina Ravera \\ Department of Economics and Business Studies, University of Genoa, Genoa, Italy \\ Email: gosio@economia.unige.it, lari@economia.unige.it, ravera@economia.unige.it
}

Received 30 April 2015; accepted 5 June 2015; published 8 June 2015

Copyright (C) 2015 by authors and Scientific Research Publishing Inc.

This work is licensed under the Creative Commons Attribution International License (CC BY). http://creativecommons.org/licenses/by/4.0/

c) (i) Open Access

\begin{abstract}
The paper deals with the optimal proportional reinsurance in a collective risk theory model involving two classes of insurance business. These classes are dependent through the number of claims. The objective of the insurer is to choose an optimal reinsurance strategy that maximizes the expected exponential utility of terminal wealth. We are able to derive the evolution of the insurer surplus process under the assumption that the number of claims of the two classes of the insurance business has a Poisson bivariate distribution. We face the problem of finding the optimal strategy using the dynamic programming approach. Therefore, we determine the infinitesimal generator for the surplus process and for the value function, and we give the Hamilton Jacobi Bellmann (HJB) equation. Under particular assumptions, we obtain explicit form of the optimal reinsurance strategy on correspondent value function.
\end{abstract}

\section{Keywords}

Collective Risk Theory, Optimal Proportional Reinsurance, Dynamic Programming

\section{Introduction}

The classical Cramer-Lundberg risk model assumes that the stochastic process $N(t)$ denotes the number of claims up to time $t$ and the random variables $X_{j}$, the claim size of the $j$-th claim. In this model, $N(t)$ is independent of the claim sizes and the claim sizes are independent and identically distributed; however, this assumption is too restrictive at times. Several authors have proposed models with dependence between the risks. Among the various types of dependence models proposed, in this paper we refer to the case where the dependency is obtained assuming that the insurer has $n \geq 2$ correlated classes of insurance business, where $N_{i}(t), i=1,2, \cdots, n$, is the number of claims of the $i$-th class; $X_{i j}, j=1,2, \cdots$, is the claim sizes of the $j$-th claim of the $i$-th class and the numbers $N_{i}(t)$ are dependent claim count processes. Models of this type are proposed 
in [1]-[6]. In [3] [4], a risk model involving two dependent classes of insurance business is considered in a given period of time and the expected utility of the insurer wealth is maximized by the determination of optimal retention limits of Unlimited or Limited Excess of Loss reinsurance.

In this paper, we consider an optimal proportional reinsurance problem of an insurer whose surplus process is generated by two dependent classes of insurance business. The objective is to choose an optimal reinsurance strategy; in order to maximize the insurer's expected exponential utility of terminal wealth we use the dynamic programming approach.

The paper is organized as follows. In Section 2, we present the risk model. In Section 3, we find the surplus evolution and the conditional expected utility of the insurer's terminal surplus, define the problem and give the corresponding value function. In Section 4, using the infinitesimal generator, we derive the HJB equation and justify the form of the value function. Finally, in Section 5, we discuss the solution giving an explicit solution in a particular framework.

\section{The Model}

In the finite time horizon $[0, T], 0<T<\infty$, we consider a model that involves two risks that may represent two classes of insurance business dependent through the number of claims. Let $\left\{N_{i}(t) ; t \in[0, T], i=1,2\right\}$, are the arrival processes of the respective claims. We denote by $X_{i j}, j=1,2, \cdots$, the random variable claim size of the risk $i, i=1,2$, assuming that these random variables have respectively the same distribution function $F_{i}$, with $F_{i}(x)=0$ for $x \leq 0$, and mean values $\mu_{i}<\infty, i=1,2$. Moreover, we assume that the moment generating function of the random variables $X_{i j}, i=1,2, j=1,2, \cdots$, exists. Finally, we assume that the random variables $\left\{X_{i j}, i=1,2, j=1,2, \cdots\right\}$, are mutually independent, and independent of $\left\{N_{i}(t) ; t \in[0, T], i=1,2\right\}$. We denote by $S_{i}(t), t \in[0, T]$, the aggregate claims amounts of the risk $i, i=1,2$. We assume that the processes $\left\{N_{i}(t) ; t \in[0, T], i=1,2\right\}$, are Poisson processes defined as follows:

$$
N_{i}(t)=Y_{i}(t)+Y_{12}(t), i=1,2, t \in[0, T]
$$

where $Y_{1}(t), Y_{2}(t), Y_{12}(t)$ are Poisson random variables that are mutually independent having positive mean, in the time unit, $\theta_{1}, \theta_{2}, \theta_{12}$ respectively. It follows that $\left\{N_{1}(t), N_{2}(t) ; t \in[0, T]\right\}$ has a Poisson bivariate distribution and that $S_{1}(t)$ and $S_{2}(t)$ are correlated by $\theta_{12}$; in fact, it results:

$$
S_{1}(t)=\sum_{j=1}^{N_{1}(t)} X_{1 j}, S_{2}(t)=\sum_{j=1}^{N_{2}(t)} X_{2 j} .
$$

In the following, we will use the variables $X_{i}, i=1,2$, identically distributed to $X_{i j}, j=1,2, \cdots$.

We denote by $c, i=1,2$ the premium rate, for the time unit, assuming that the premium calculation principle is the expected value principle with loading coefficient $\eta_{i}$, that is:

$$
c_{i}=\left(1+\eta_{i}\right)\left(\theta_{i}+\theta_{12}\right) \mu_{i}, \eta_{i}>0, \quad i=1,2 .
$$

We introduce a proportional reinsurance: the reinsurer pays $\left(1-a_{i}\right), a_{i} \in[0,1]$, of each claim of the type $i, i$ $=1,2$ and he receives from the insurer the reinsurance premium. We denote by $P_{i}\left(a_{i}\right), i=1,2$, the reinsurance premium and we get

$$
P_{i}\left(a_{i}\right)=\left(1-a_{i}\right)\left(1+\gamma_{i}\right)\left(\theta_{i}+\theta_{12}\right) \mu_{i}:=\left(1-a_{i}\right) P_{i} ; \gamma_{i}>\eta_{i}, \quad i=1,2
$$

for which it is

$$
P_{i}=\left(1+\gamma_{i}\right)\left(\theta_{i}+\theta_{12}\right) \mu_{i}>c_{i}, \quad i=1,2 .
$$

Note that the condition $c_{i}-\left(1-a_{i}\right) P_{i}>a_{i}\left(\theta_{i}+\theta_{12}\right) \mu_{i}, i=1,2$ linked to the positivity of the security loading is not assumed, as it may or may not be satisfied.

\section{The Problem}

We denote by $\left(1-a_{i}(t)\right), a_{i}(t) \in[0,1]$ the proportion insured at time $t, t \in[0, T]$, where $a_{i}(t)$ is therefore the risk exposure of insurer at time $t$. We assume that, at every time $t, t \in[0, T]$, the insurer can choose the risk exposure $a_{i}(t) \in[0,1]$, according to the observable information about the insurance risk processes up to time $t$. There- 
fore $a_{i}(t), i=1,2$, are the insurer's control parameters; let $A:=\left\{\alpha(t)=\left(a_{1}(t), a_{2}(t)\right) ; 0 \leq a_{i}(t) \leq 1, i=1,2\right\}$ be the set of all admissible policies. The objective for the insurer is to choose an optimal reinsurance strategy that maximize the expected exponential utility of terminal wealth. We will make use of the HJB theory to face the problem. After the reinsurance the total claim amount charged to the insurer is

$$
\bar{S}_{1}(t)+\bar{S}_{2}(t) \text {, being } \bar{S}_{i}(t)=\sum_{j=1}^{N_{i}(t)} X_{i j} a_{i}, i=1,2 \text {. }
$$

We denote by $R(t, \alpha)$ the surplus process of the insurer adopting the reinsurance strategy process $\{\alpha(t) ; t \in[0, T]\}$. The surplus evolves over time as:

$$
\mathrm{d} R(t, \alpha)=\left[c_{1}-\left(1-a_{1}\right) P_{1}+c_{2}-\left(1-a_{2}\right) P_{2}\right] \mathrm{d} t-\mathrm{d}\left[\bar{S}_{1}(t)+\bar{S}_{2}(t)\right]:=P \mathrm{~d} t-\mathrm{d}\left[\bar{S}_{1}(t)+\bar{S}_{2}(t)\right]
$$

We recall that $N_{1}(t)$ and $N_{2}(t)$ are defined by (1), then the process $\left\{\left(N_{1}(t), N_{2}(t)\right) ; t \in[0, T]\right\}$ has a bivariate Poisson distribution. This fact, noting that the process has stationary increments (see [7]) and using results in [8], allows us to determine the following joint probabilities:

$$
\begin{aligned}
& \operatorname{Pr}\left[N_{1}(t+\mathrm{d} t)-N_{1}(t)=0 \text { and } N_{2}(t+\mathrm{d} t)-N_{2}(t)=0 \mid N_{1}(s) \text { and } N_{2}(s) \forall s \leq t\right] \\
& =\mathrm{e}^{-\left(\theta_{1}+\theta_{2}+\theta_{12}\right) \mathrm{d} t} \sum_{i=0}^{0} \frac{\left(\theta_{1} \mathrm{~d} t\right)^{0-i}\left(\theta_{2} \mathrm{~d} t\right)^{0-i}\left(\theta_{12} \mathrm{~d} t\right)^{i}}{(0-i) !(0-i) ! i !} \\
& =\mathrm{e}^{-\left(\theta_{1}+\theta_{2}+\theta_{12}\right) \mathrm{d} t} \cong 1-\left(\theta_{1}+\theta_{2}+\theta_{12}\right) \mathrm{d} t \\
& \operatorname{Pr}\left[N_{1}(t+\mathrm{d} t)-N_{1}(t)=1 \text { and } N_{2}(t+\mathrm{d} t)-N_{2}(t)=0 \mid N_{1}(s) \text { and } N_{2}(s) \forall s \leq t\right] \\
& =\mathrm{e}^{-\left(\theta_{1}+\theta_{2}+\theta_{12}\right) \mathrm{d} t} \sum_{i=0}^{0} \frac{\left(\theta_{1} \mathrm{~d} t\right)^{1-i}\left(\theta_{2} \mathrm{~d} t\right)^{0-i}\left(\theta_{12} \mathrm{~d} t\right)^{i}}{(1-i) !(0-i) ! i !} \\
& =\mathrm{e}^{-\left(\theta_{1}+\theta_{2}+\theta_{12}\right) \mathrm{dt}} \theta_{1} \mathrm{~d} t \cong\left(1-\left(\theta_{1}+\theta_{2}+\theta_{12}\right) \mathrm{d} t\right) \theta_{1} \mathrm{~d} t \cong \theta_{1} \mathrm{~d} t ; \\
& \operatorname{Pr}\left[N_{1}(t+\mathrm{d} t)-N_{1}(t)=0 \text { and } N_{2}(t+\mathrm{d} t)-N_{2}(t)=1 \mid N_{1}(s) \text { and } N_{2}(s) \forall s \leq t\right] \\
& =\mathrm{e}^{-\left(\theta_{1}+\theta_{2}+\theta_{12}\right) \mathrm{d} t} \theta_{2} \mathrm{~d} t \cong \theta_{2} \mathrm{~d} t ; \\
& \operatorname{Pr}\left[N_{1}(t+\mathrm{d} t)-N_{1}(t)=1 \text { and } N_{2}(t+\mathrm{d} t)-N_{2}(t)=1 \mid N_{1}(s) \text { and } N_{2}(s) \forall s \leq t\right] \\
& =\mathrm{e}^{-\left(\theta_{1}+\theta_{2}+\theta_{12}\right) \mathrm{d} t} \sum_{i=0}^{1} \frac{\left(\theta_{1} \mathrm{~d} t\right)^{1-i}\left(\theta_{2} \mathrm{~d} t\right)^{1-i}\left(\theta_{12} \mathrm{~d} t\right)^{i}}{(1-i) !(1-i) ! i !} \\
& =\mathrm{e}^{-\left(\theta_{1}+\theta_{2}+\theta_{12}\right) \mathrm{d} t}\left[\theta_{1} \theta_{2}(\mathrm{~d} t)^{2}+\theta_{12} \mathrm{~d} t\right] \cong\left(1-\left(\theta_{1}+\theta_{2}+\theta_{12}\right) \mathrm{d} t\right) \theta_{12} \mathrm{~d} t \cong \theta_{12} \mathrm{~d} t .
\end{aligned}
$$

From previous result it follows

$$
\begin{aligned}
& {\left[\bar{S}_{1}(t+\mathrm{d} t)+\bar{S}_{2}(t+\mathrm{d} t)\right]-\left[\bar{S}_{1}(t)+\bar{S}_{2}(t)\right]} \\
& =\left\{\begin{array}{l}
0 \text { with probability } 1-\left(\theta_{1}+\theta_{2}+\theta_{12}\right) \mathrm{d} t \\
a_{1} X_{1} \text { with probability } \theta_{1} \mathrm{~d} t \\
a_{2} X_{2} \text { with probability } \theta_{2} \mathrm{~d} t \\
a_{1} X_{1}+a_{2} X_{2} \text { with probability } \theta_{12} \mathrm{~d} t
\end{array}\right.
\end{aligned}
$$

where $X_{i}$ are identically distributed to $X_{i j}, \quad i=1,2, j=1,2, \cdots$.

We consider an utility function $u: R \rightarrow R, u \in C^{2}(R)$, strictly increasing and concave (that is $u^{\prime}>0$ and $\left.u^{\prime \prime}<0\right)$. For each control strategy $\alpha=\left(a_{1}, a_{2}\right), \quad \alpha \in A$, given $R(t, \alpha)=x, t \in[0, T]$, we define the following conditional expected utility of the insurer's terminal surplus:

$$
V^{\alpha}(t, x):=E[u(R(T, \alpha)) \mid R(t, \alpha)=x], t \in[0, T], \alpha \in A .
$$

As previously stated, the insurer's goal is to determine an optimal reinsurance strategy $\alpha \in A$ so as to maximize the expected utility of the terminal surplus (8). We therefore consider the following problem 


$$
V(t, x)=\sup _{\alpha \in A} V^{\alpha}(t, x) .
$$

It follows that the insurer has to find the optimal strategy $\alpha^{*} \in A$ and the corresponding value function $V(t, x)$ such that:

$$
V(t, x)=V^{\alpha^{*}}(t, x)
$$

with the usual boundary condition (see [9] [10])

$$
V(T, x)=u(x) .
$$

\section{The HJB Equation and the Value Function}

We can find the infinitesimal generator for the process $R(t, \alpha)$ and for the function $V$. The procedure is similar to that one used in [11] and in [12].

Theorem 1. Let $V(t, x)$ be defined by (9) and (10) and let $V(t, x) \in C^{1,1}([0, T] \times R)$. Therefore, $V$ satisfies the following HJB equation:

$$
\begin{aligned}
0= & \sup _{\propto \in A}\left\{\partial V(t, x) / \partial t+\left[c_{1}-\left(1-a_{1}\right) P_{1}+c_{2}-\left(1-a_{2}\right) P_{2}\right] \partial V(t, x) / \partial x\right\} \\
& +\theta_{1} \int_{0}^{+\infty}\left[V\left(t, x-a_{1} x_{1}\right)-V(t, x)\right] \mathrm{d} F_{1}\left(x_{1}\right) \\
& +\theta_{2} \int_{0}^{+\infty}\left[V\left(t, x-a_{2} x_{2}\right)-V(t, x)\right] \mathrm{d} F_{2}\left(x_{2}\right) \\
& +\theta_{12} \int_{0}^{+\infty} \int_{0}^{+\infty}\left[V\left(t, x-a_{1} x_{1}-a_{2} x_{2}\right)-V(t, x)\right] \mathrm{d} F_{1}\left(x_{1}\right) \mathrm{d} F_{2}\left(x_{2}\right), \alpha=\left(\alpha_{1}, \alpha_{2}\right)
\end{aligned}
$$

Proof. We derive the following infinitesimal generator for the process $R(t, \alpha)$ and for the function $V$ :

$$
L_{t} V(t, x)=\lim _{h \searrow 0} 1 / h E[V(t+h, R(t+h, \alpha))-V(t, R(t, \alpha)) \mid R(t, \alpha)=x]
$$

it allows us to write the HJB Equation (12).

We recall that, by (6) and (7) it results in

$$
\begin{aligned}
& V(t+h, R(t+h, \alpha)) \\
& =\left\{\begin{array}{l}
V(t+h, R(t, \alpha)+P h) \text { with probability } 1-\left(\theta_{1}+\theta_{2}+\theta_{12}\right) h \\
V\left(t+h, R(t, \alpha)+P h-a_{1} X_{1}\right) \text { with probability } \theta_{1} h \\
V\left(t+h, R(t, \alpha)+P h-a_{2} X_{2}\right) \text { with probability } \theta_{2} h \\
V\left(t+h, R(t, \alpha)+P h-a_{1} X_{1}-a_{2} X_{2}\right) \text { with probability } \theta_{12} h
\end{array}\right.
\end{aligned}
$$

therefore we have, remembering the independence between $X_{i}$ and $N_{i}, i=1,2$ :

$$
\begin{aligned}
& \lim _{h \backslash 0} 1 / h E[V(t+h, R(t+h, \alpha))-V(t, R(t, \alpha)) \mid R(t, \alpha)=x] \\
& =\lim _{h \searrow 0}\left\{(V(t+h, x+P h)-V(t, x)) / h-\left(\theta_{1}+\theta_{2}+\theta_{12}\right) V(t+h, x+P h)-V(t, x)\right\} \\
& \quad+\lim _{h \searrow 0} E\left[\left\{V\left(t+h, x+P h-a_{1} X_{1}\right)-V(t, x)\right\} \theta_{1}+\left\{V\left(t+h, x+P h-a_{2} X_{2}\right)-V(t, x)\right\} \theta_{2}\right. \\
& \left.\quad+\left\{V\left(t+h, x+P h-a_{1} X_{1}-a_{2} X_{2}\right)-V(t, x)\right\} \theta_{12}\right] \\
& =\lim _{\sqrt{1+P^{2}} h:=\bar{h} \searrow 0} \frac{V\left(t+\frac{\bar{h}}{\sqrt{1+P^{2}}}, x+\frac{\bar{h} P}{\sqrt{1+P^{2}}}-V(t, x)\right)}{\bar{h}} \sqrt{1+P^{2}} \\
& \quad+\theta_{1} \int_{0}^{+\infty}\left[V\left(t, x-a_{1} x_{1}\right)-V(t, x)\right] \mathrm{d} F_{1}\left(x_{1}\right)+\theta_{2} \int_{0}^{+\infty}\left[V\left(t, x-a_{2} x_{2}\right)-V(t, x)\right] \mathrm{d} F_{2}\left(x_{2}\right) \\
& \quad+\theta_{12} \int_{0}^{+\infty} \int_{0}^{+\infty}\left[V\left(t, x-a_{1} x_{1}-a_{2} x_{2}\right)-V(t, x)\right] \mathrm{d} F_{1}\left(x_{1}\right) \mathrm{d} F_{2}\left(x_{2}\right) .
\end{aligned}
$$


Therefore $V$ must satisfy Equation (12).

We introduce the following utility function

$$
u(x)=-1 / \beta^{\mathrm{e}^{-\beta x}}, \beta>0, x \in R
$$

With the purpose to write (9) we observe that:

1) from (6) and remembering that Poisson processes have stationary increments, we obtain:

$$
[R(T, \alpha) \mid R(t, \alpha)=x]=x+(T-t) P-\left[\bar{S}_{1}(T-t)+\bar{S}_{2}(T-t)\right]
$$

with, as previously stated, $\left(P=c_{1}-\left(1-a_{1}\right) P_{1}+c_{2}-\left(1-a_{2}\right) P_{2}\right)$;

2) in Section 2 we have assumed that the moment generating functions of random variables $X_{i j}$, $i=1,2, j=1,2, \cdots$, and therefore of random variables $X_{i}, i=1,2$, exist.

We denote those functions by $M_{X_{i}}, i=1,2$, and we observe that $M_{X_{i}}\left(\alpha_{i} \beta\right)=M_{a_{i} X_{i}}(\beta)$;

3 ) according to [7] and from the probability generating function of the bivariate Poisson distribution (see [8], p. 126), it results in:

$$
\begin{aligned}
& E\left[\mathrm{e}^{\beta\left[\bar{S}_{1}(T-t)+\bar{S}_{2}(T-t)\right]}\right]=E\left[E\left[\mathrm{e}^{\beta\left[\bar{S}_{1}(T-t)+\bar{S}_{2}(T-t)\right] \mid N_{1}(T-t) \text { and } N_{2}(T-t)}\right]\right] \\
& =E\left[\left(M_{a_{1} X_{1}}(\beta)\right)^{N_{1}(T-t)}\left(M_{a_{2} X_{2}}(\beta)\right)^{N_{2}(T-t)}\right] \\
& =\mathrm{e}^{\left[\left(\theta_{1}+\theta_{12}\right)\left(M_{a_{1} X_{1}}(\beta)-1\right)+\left(\theta_{2}+\theta_{12}\right)\left(M_{a_{2} X_{2}}(\beta)-1\right)+\theta_{12}\left(M_{a_{1} X_{1}}(\beta)-1\right)\left(M_{a_{2} X_{2}}(\beta)-1\right)\right](T-t)} \\
& :=\mathrm{e}^{(T-t) Q}
\end{aligned}
$$

4) from the previous considerations, we have:

$$
\begin{aligned}
& V^{\alpha}(t, x)=E[u(R(T, \alpha)) \mid R(t, \alpha)=x] \\
& =E\left[-\frac{1}{\beta} \mathrm{e}^{-\beta\left\{x+(T-t) P-\left[\bar{S}_{1}(T-t)+\bar{S}_{2}(T-t)\right]\right\}}\right] \\
& =\frac{1}{\beta} \mathrm{e}^{-\beta x-\beta(T-t)\left(P-\frac{Q}{\beta}\right)}
\end{aligned}
$$

Because of these considerations, we assume that the value function $V$, defined by (10) with the condition (11) has the form

$$
V(t, x)=-\frac{1}{\beta} \mathrm{e}^{-\beta[x-B(t)]}
$$

with the condition

$$
B(T)=0 .
$$

\section{Possible Solutions}

We consider the assumptions (18); it results in:

$$
\begin{gathered}
\frac{\partial V}{\partial t} V(t, x)=\beta B^{\prime}(t) V(t, x), \\
\frac{\partial V}{\partial x} V(t, x)=-\beta V(t, x), \\
V(t, x-z)-V(t, x)=\left(\mathrm{e}^{\beta z}-1\right) V(t, x) .
\end{gathered}
$$

Therefore, (12) becomes 


$$
\begin{aligned}
0= & \sup _{\propto \in A}\left\{\beta B^{\prime}(t) V(t, x)-\left[c_{1}-\left(1-a_{1}\right) P_{1}+c_{2}-\left(1-a_{2}\right) P_{2}\right] \beta V(t, x)\right. \\
& +\theta_{1} \int_{0}^{+\infty}\left(\mathrm{e}^{\beta a_{1} x_{1}}-1\right) \mathrm{d} F_{1}\left(x_{1}\right) V(t, x)+\theta_{2} \int_{0}^{+\infty}\left(\mathrm{e}^{\beta a_{2} x_{2}}-1\right) \mathrm{d} F_{2}\left(x_{2}\right) V(t, x) \\
& \left.+\theta_{12} \int_{0}^{+\infty} \int_{0}^{+\infty}\left(\mathrm{e}^{\beta\left(a_{1} x_{1}+a_{2} x_{2}\right)}-1\right) \mathrm{d} F_{1}\left(x_{1}\right) \mathrm{d} F_{2}\left(x_{2}\right) V(t, x)\right\}, \alpha=\left(\alpha_{1}, \alpha_{2}\right)
\end{aligned}
$$

with $B(T)=0$; hence, remembering that $-\beta V(t, x)>0$, it results in:

$$
\begin{aligned}
0= & \sup _{\propto \in A}\left\{-B^{\prime}(t)+\left[c_{1}-\left(1-a_{1}\right) P_{1}+c_{2}-\left(1-a_{2}\right) P_{2}\right]\right. \\
& -\theta_{1} / \beta \int_{0}^{+\infty}\left(\mathrm{e}^{\beta a_{1} x_{1}}-1\right) \mathrm{d} F_{1}\left(x_{1}\right)-\theta_{2} / \beta \int_{0}^{+\infty}\left(\mathrm{e}^{\beta a_{2} x_{2}}-1\right) \mathrm{d} F_{2}\left(x_{2}\right) \\
& \left.-\theta_{12} / \beta \int_{0}^{+\infty} \int_{0}^{+\infty}\left(\mathrm{e}^{\beta\left(a_{1} x_{1}+a_{2} x_{2}\right)}-1\right) \mathrm{d} F_{1}\left(x_{1}\right) \mathrm{d} F_{2}\left(x_{2}\right)\right\}, \alpha=\left(\alpha_{1}, \alpha_{2}\right)
\end{aligned}
$$

with $B(T)=0$.

Assuming the particular case where the insurer's risk exposure is the same for the two classes of the insurance business; that is $a_{i}=a, i=1,2,0 \leq a \leq 1$; the control parameter is $a$ (resulting in $\alpha=(a, a)$ ), and (20) becomes:

$$
\begin{aligned}
0= & \sup _{a \in[0,1]}\left\{-B^{\prime}(t)+\left[c_{1}+c_{2}-(1-a)\left(P_{1}+P_{2}\right)\right]\right. \\
& -\theta_{1} / \beta \int_{0}^{+\infty}\left(\mathrm{e}^{\beta a x_{1}}-1\right) \mathrm{d} F_{1}\left(x_{1}\right)-\theta_{2} / \beta \int_{0}^{+\infty}\left(\mathrm{e}^{\beta a x_{2}}-1\right) \mathrm{d} F_{2}\left(x_{2}\right) \\
& \left.-\theta_{12} / \beta \int_{0}^{+\infty} \int_{0}^{+\infty}\left(\mathrm{e}^{\beta a\left(x_{1}+x_{2}\right)}-1\right) \mathrm{d} F_{1}\left(x_{1}\right) \mathrm{d} F_{2}\left(x_{2}\right)\right\}
\end{aligned}
$$

with $B(T)=0$. The problem of the determination of the optimal control $a^{*}$ can be easily solved, as we will see in the following.

For simplicity, we write (21) as follows

$$
0=\sup _{a \in[0,1]} G(a)
$$

observing that

$$
\begin{aligned}
G^{\prime}(a)= & \left(P_{1}+P_{2}\right)-\theta_{1} \int_{0}^{+\infty} x_{1} \mathrm{e}^{\beta a x_{1}} \mathrm{~d} F_{1}\left(x_{1}\right)-\theta_{2} \int_{0}^{+\infty} x_{2} \mathrm{e}^{\beta a x_{2}} \mathrm{~d} F_{2}\left(x_{2}\right) \\
& -\theta_{12} \int_{0}^{+\infty} \int_{0}^{+\infty}\left(x_{1}+x_{2}\right) \mathrm{e}^{\beta a\left(x_{1}+x_{2}\right)} \mathrm{d} F_{1}\left(x_{1}\right) \mathrm{d} F_{2}\left(x_{2}\right)
\end{aligned}
$$

from which we obtain:

1) $G^{\prime}(0)=\left(P_{1}+P_{2}\right)-\theta_{1} \mu_{1}-\theta_{2} \mu_{2}-\theta_{12}\left(\mu_{1}+\mu_{2}\right)=P_{1}-\mu_{1}\left(\theta_{1}+\theta_{12}\right)+P_{2}-\mu_{2}\left(\theta_{2}+\theta_{12}\right)>0$ by (3) and (4), from which we deduce that $G(a)$ is an increasing function in $a=0$ and therefore $a^{*} \neq 0$;

2) $G^{\prime \prime}(a)=-\theta_{1} \beta \int_{0}^{+\infty} x_{1}^{2} \mathrm{e}^{\beta a x_{1}} \mathrm{~d} F_{1}\left(x_{1}\right)-\theta_{2} \beta \int_{0}^{+\infty} x_{2}^{2} \mathrm{e}^{\beta a x_{2}} \mathrm{~d} F_{2}\left(x_{2}\right)$

$-\theta_{12} \beta \int_{0}^{+\infty} \int_{0}^{+\infty}\left(x_{1}+x_{2}\right)^{2} \mathrm{e}^{\beta a\left(x_{1}+x_{2}\right)} \mathrm{d} F_{1}\left(x_{1}\right) \mathrm{d} F_{2}\left(x_{2}\right)<0$;

3) $\lim _{a \rightarrow+\infty} G^{\prime}(a)=-\infty$.

From the previous results it follows that $G^{\prime}(a)$ is equal to zero in a single point $\tilde{a} \in(0,+\infty)$ and:

if $G^{\prime}(1)>0 \rightarrow \tilde{a}>1$ and therefore $a^{*}=1$;

if $G^{\prime}(1)<0 \rightarrow \tilde{a} \in(0,1)$ and therefore $a^{*}=\tilde{a} \in(0,1)$;

if $G^{\prime}(1)=0 \rightarrow \tilde{a} \in 1$ and therefore $a^{*}=1$

being

$$
\begin{aligned}
G^{\prime}(1)= & \left(P_{1}+P_{2}\right)-\theta_{1} \int_{0}^{+\infty} x_{1} \mathrm{e}^{\beta x_{1}} \mathrm{~d} F_{1}\left(x_{1}\right)-\theta_{2} \int_{0}^{+\infty} x_{2} \mathrm{e}^{\beta x_{2}} \mathrm{~d} F_{2}\left(x_{2}\right) \\
& -\theta_{12} \int_{0}^{+\infty} \int_{0}^{+\infty}\left(x_{1}+x_{2}\right) \mathrm{e}^{\beta\left(x_{1}+x_{2}\right)} \mathrm{d} F_{1}\left(x_{1}\right) \mathrm{d} F_{2}\left(x_{2}\right) .
\end{aligned}
$$

We therefore obtain the following results.

If $G^{\prime}(1) \geq 0$, it results in $a^{*}=1$ and by (21) we have: 


$$
\begin{aligned}
0=G(1)= & -B^{\prime}(t)+c_{1}+c_{2}-\frac{\theta_{1}}{\beta} \int_{0}^{+\infty}\left(\mathrm{e}^{\beta x_{1}}-1\right) \mathrm{d} F_{1}\left(x_{1}\right) \\
& -\frac{\theta_{2}}{\beta} \int_{0}^{+\infty}\left(\mathrm{e}^{\beta x_{2}}-1\right) \mathrm{d} F_{2}\left(x_{2}\right)-\frac{\theta_{12}}{\beta} \int_{0}^{+\infty} \int_{0}^{+\infty}\left(\mathrm{e}^{\beta\left(x_{1}+x_{2}\right)}-1\right) \mathrm{d} F_{1}\left(x_{1}\right) \mathrm{d} F_{2}\left(x_{2}\right)
\end{aligned}
$$

with $B(T)=0$, from which we obtain

$$
\begin{aligned}
B(t)=- & {\left[c_{1}+c_{2}-\frac{\theta_{1}}{\beta} \int_{0}^{+\infty}\left(\mathrm{e}^{\beta x_{1}}-1\right) \mathrm{d} F_{1}\left(x_{1}\right)-\frac{\theta_{2}}{\beta} \int_{0}^{+\infty}\left(\mathrm{e}^{\beta x_{2}}-1\right) \mathrm{d} F_{2}\left(x_{2}\right)\right.} \\
& \left.-\frac{\theta_{12}}{\beta} \int_{0}^{+\infty} \int_{0}^{+\infty}\left(\mathrm{e}^{\beta\left(x_{1}+x_{2}\right)}-1\right) \mathrm{d} F_{1}\left(x_{1}\right) \mathrm{d} F_{2}\left(x_{2}\right)\right](T-t)
\end{aligned}
$$

then the resulting value function (18) is:

$$
V(t, x)=-1 / \beta^{\mathrm{e}^{-\beta[x-B(t)]}} \text {, where } B(t) \text { is given by (24). }
$$

If $G^{\prime}(1)<0$, it results in $a^{*}=\tilde{a}$ such that $G^{\prime}(\tilde{a})=0$. Therefore, remembering (22), it results in

$$
\begin{aligned}
\left(P_{1}+P_{2}\right)= & \theta_{1} \int_{0}^{+\infty} x_{1} \mathrm{e}^{\beta \tilde{a} x_{1}} \mathrm{~d} F_{1}\left(x_{1}\right)+\theta_{2} \int_{0}^{+\infty} x_{2} \mathrm{e}^{\beta \tilde{a} x_{2}} \mathrm{~d} F_{2}\left(x_{2}\right) \\
& +\theta_{12} \int_{0}^{+\infty} \int_{0}^{+\infty}\left(x_{1}+x_{2}\right) \mathrm{e}^{\beta \tilde{a}\left(x_{1}+x_{2}\right)} \mathrm{d} F_{1}\left(x_{1}\right) \mathrm{d} F_{2}\left(x_{2}\right)
\end{aligned}
$$

and by (21):

$$
\begin{aligned}
0=G(\tilde{a})= & -B^{\prime}(t)+c_{1}+c_{2}-(1-\tilde{a})\left(P_{1}+P_{2}\right) \\
& -\frac{\theta_{1}}{\beta} \int_{0}^{+\infty}\left(\mathrm{e}^{\beta \tilde{a} x_{1}}-1\right) \mathrm{d} F_{1}\left(x_{1}\right)-\frac{\theta_{2}}{\beta} \int_{0}^{+\infty}\left(\mathrm{e}^{\beta \tilde{\alpha} x_{2}}-1\right) \mathrm{d} F_{2}\left(x_{2}\right) \\
& -\frac{\theta_{12}}{\beta} \int_{0}^{+\infty} \int_{0}^{+\infty}\left(\mathrm{e}^{\beta \tilde{a}\left(x_{1}+x_{2}\right)}-1\right) \mathrm{d} F_{1}\left(x_{1}\right) \mathrm{d} F_{2}\left(x_{2}\right)
\end{aligned}
$$

with $B(T)=0$. From the previous, with a procedure analogous to that followed in the case $a^{*}=1$, it is possible to find $B(t)$ and the resulting value function.

\section{Acknowledgements}

We thank the Editor and the Referees for their comments.

\section{References}

[1] Ambagaspitiya, R.S. (1998) Compound Bivariate Lagrange Poisson Distributions. Insurance: Mathematics and Economics, 23, 21-31. http://dx.doi.org/10.1016/S0167-6687(98)00020-1

[2] Ambagaspitiya, R.S. (1998) On the Distribution of a Sum of Correlated Aggregate Claims. Insurance: Mathematics and Economics, 23, 15-19. http://dx.doi.org/10.1016/s0167-6687(98)00018-3

[3] Centeno, M.L. (2005) Dependent Risks and Excess of Loss Reinsurance. Insurance: Mathematics and Economics, 37, 229-238. http://dx.doi.org/10.1016/j.insmatheco.2004.12.001

[4] Gosio, C., Lari, E.C. and Ravera, M. (2013) Optimal Expected Utility of the Wealth for Two Dependent Classes of Insurance Business. Theoretical Economics Letters, 3, 90-95. http://dx.doi.org/10.4236/tel.2013.32015

[5] Yuen, K.C., Guo, J. and Wu, X. (2002) On Correlated Aggregate Claims Model with Poisson and Erlang Risk Processes. Insurance: Mathematics and Economics, 31, 205-214. http://dx.doi.org/10.1016/s0167-6687(02)00150-6

[6] Yuen, K.C., Guo, J. and Wu, X. (2006) On the First Time of Ruine in the Bivariate Compound Poisson Model. Insurance: Mathematics and Economics, 38, 298-308. http://dx.doi.org/10.1016/j.insmatheco.2005.08.011

[7] Bowers, N.L., Gerber, H.U., Hickman, J.C., Jones, D.A. and Nesbitt, C.J. (1986) Actuarial Mathematics. Society of Actuaries, Itaxa, Illinois.

[8] Johnson, N.L. and Balakrishnan, N. (1997) Discrete Multivariate Distributions. Wiley \& Sons, Hoboken.

[9] Lin, X. and Li, Y. (2011) Optimal Reinsurance and Investment for a Jump Diffusion Risk Process under the CEV 
Model. North American Actuarial Journal, 15, 417-431. http://dx.doi.org/10.1080/10920277.2011.10597628

[10] Pham, H. (2010) Continuous-Time Stochastic Control and Optimization Problem with Financial Applications. Springer Verlag, Berlin.

[11] Çetin, U. (2014) An Introduction to Markov Processes and Their Applications in Mathematical Economics. Lecture Notes, Department of Statistic, London School of Economics and Political Science, London.

[12] Hipp, C. (2004) Stochastic Control with Applications in Insurance, Stochastic Methods in Finance. Lecture Notes in Mathematics, 1856, 127-164. 